\title{
Tumor-suppressor activity of RRIG1 in breast cancer
}

\author{
Guihong Zhang ${ }^{1,3}$, Abenaa Brewster ${ }^{1}$, Baoxiang Guan ${ }^{1}$, Zhen Fan², Powel H Brown ${ }^{1}$, Xiao-Chun Xu ${ }^{1,3^{*}}$
}

\begin{abstract}
Background: Retinoid receptor-induced gene-1 (RRIG1) is a novel gene that has been lost in several types of human cancers. The aim of this study was to determine whether RRIG1 plays a role in breast cancer, such as in the suppression of breast cancer cell growth and invasion.

Methods: Immunohistochemistry was used to detect RRIG1 expression in breast tissue specimens. Gene transfection was used to restore or knock down RRIG1 expression in breast cancer cell lines for analysis of cell viability, colony formation, and migration/invasion potential. Reverse-transcription polymerase chain reaction and western blot assays were used to detect the changes in gene expression. The RhoA activation assay was used to assess RRIG1-induced inhibition of RhoA activity.
\end{abstract}

Results: The immunohistochemical data showed that RRIG1 expression was reduced in breast cancer tissues compared with normal and atypical hyperplastic breast tissues. RRIG1 expression was inversely correlated with lymph node metastasis of breast cancer but was not associated with the status of hormone receptors, such as estrogen receptor, progesterone receptor, or HER2. Furthermore, restoration of RRIG1 expression inhibited proliferation, colony formation, migration, and invasion of breast cancer cells. Expression of RRIG1 also reduced phosphorylated Erk1/2 and Akt levels; c-Jun, MMP9, and Akt expressions; and RhoA activity. In contrast, knockdown of RRIG1 expression promoted breast cancer cell proliferation, colony formation, migration, and invasion potential.

Conclusion: The data from the current study indicated that RRIG1 expression was reduced or lost in breast cancer and that restoration of RRIG1 expression suppressed breast cancer cell growth and invasion capacity. Future studies will determine the underlying molecular mechanisms and define RRIG1 as a tumor-suppressor gene in breast cancer.

\section{Background}

Breast cancer is the leading cause of cancer-related death in women between 35 and 45 years of age and remains the second-leading cause of cancer-related death among all women in the United States [1]. Despite success in screening for early stages of breast cancer and remarkable improvement in treatment outcomes, many women still develop metastatic disease and ultimately die [2-4]. Efforts for ultimate elimination of this disease should include an emphasis on 1) a better understanding of breast cancer biology, including elucidation of the functions of the genes involved in breast cancer development, progression, and metastasis; 2) development of

\footnotetext{
* Correspondence: xxu@mdanderson.org

'Department of Clinical Cancer Prevention, The University of Texas M. D.

Anderson Cancer Center, Houston, Texas 77030, USA

Full list of author information is available at the end of the article
}

novel biomarkers for early detection, pretreatment staging, prediction of response to treatments, monitoring disease progression, and prognosis of breast cancer; and 3) innovative approaches for treatment and prevention of breast cancer.

Our group recently identified and cloned a novel retinoid receptor-induced gene, RRIG1 [5-8]. We found that the RRIG1 gene covers $4.181 \mathrm{~kb}$ of genomic sequences and is localized at chromosome 9q34 with 6 exons, coding a protein with 276 amino acids. RRIG1 mRNA is expressed in a broad range of normal tissues, but its expression is lost in various types of cancers, including breast cancer [5,6]. RRIG1 mediates the effect of RAR- $\beta_{2}$ on gene expression (e.g., Erk $1 / 2$ and COX-2) and cell growth. RRIG1 expression was found to be correlated with tumor differentiation but inversely correlated with lymph node metastasis of esophageal cancer [6].
C Biomed Central

(C) 2011 Zhang et al; licensee BioMed Central Ltd. This is an Open Access article distributed under the terms of the Creative Commons Attribution License (http://creativecommons.org/licenses/by/2.0), which permits unrestricted use, distribution, and reproduction in any medium, provided the original work is properly cited. 
Furthermore, the transient and stable transfection of a RRIG1 expression vector resulted in growth inhibition of esophageal and prostate cancer cells $[5,6,8]$. Esophageal cancer cells transfected with RRIG1 also showed reduced tumorigenicity in nude mice [6]. These data strongly indicate that RRIG1 plays an important role in suppressing the development or progression of human cancers $[5,6,8]$. At the level of signal transduction, expression of RRIG1 inhibited Src phosphorylation and RhoA activation, which is believed to be causally linked to reduced colony formation, invasion, and proliferation in esophageal and prostatic cancer cells. In contrast, transfection of antisense RRIG1 increased RhoA activity and f-actin formation and led to increased colony formation, invasion, and proliferation in these cells $[5,8]$.

In this study, we first determined the correlation of RRIG1 expression in breast tissue specimens with the clinicopathologic characteristics of the patients and then examined the effect of RRIG1 expression on breast cancer growth and invasion. We also explored the changes in expression and phosphorylation or activation of several relevant proteins following experimental elevation or knockdown of RRIG1 expression in breast cancer cells. Our data indicate that RRIG1 may function as a tumor-suppressor gene in breast cancer.

\section{Methods}

\section{Immunohistochemistry}

Paraffin blocks from breast tissue specimens were obtained from the Department of Pathology, Anhui Medical University, Hefei, China, guided by a protocol (\#Lab08-015) approved by the institutional review board. Patients gave consent for the use of their tissue specimens in this study. These tissue specimens consisted of samples from 15 normal mammary glands, 9 atypical hyperplasia lesions, 10 cases of ductal carcinoma in situ, and 77 cases of invasive breast cancer. For immunohistochemical analysis, these paraffin sections together with paraffin sections from the organotypic cultures were prepared and stained for RRIG1 as previously reported [6]. Briefly, the tissue sections were deparaffinized twice in xylene for $10 \mathrm{~min}$ each and rehydrated in a series of ethanol (100\%-50\%) and then subjected to antigen retrieval with $0.01 \mathrm{M}$ citric buffer in a pressure cooker for $5 \mathrm{~min}$, followed by blocking of tissue endogenous peroxidase activity with $\mathrm{H}_{2} \mathrm{O}_{2}$ treatment. The processed tissue sections were then incubated with 100 $\mu \mathrm{L}$ of $20 \%$ normal goat serum in phosphate-buffered saline (PBS) and anti-RRIG1 antibody (custom-made by Lampire Biological Laboratories, Pipersville, PA, with the CAADGLRKPQVHSARAL peptide as the antigen) at 1:500 dilutions with PBS overnight. The next day, the sections were gently washed three times with PBS and once with PBS containing $0.1 \%$ Tween 20 . They were then subjected to sequential incubations with a second antibody (goat anti-rabbit IgG; Vector Laboratories, Burlingame, $\mathrm{CA}$ ) and the $\mathrm{ABC}$ solution (in the dark) for 30 min each, with washing between incubations to remove unbound antibodies. The sections were subject to color development with 9-ethylcarbazol-3-amine for $15 \mathrm{~min}$ and counterstained with hematoxylin for $1 \mathrm{~min}$. After being covered with a cover slip, the sections were viewed and scored under a microscope. A semiquantitative scoring system was used to score both the staining intensity and the percentage of staining in the tissue sections. The staining intensity was scored as follows: 0, no staining; +, weak staining; ++ positive staining; and + ++ , very strong staining. The percentage of staining was scored as follows: 0 , no staining; + , less than $10 \%$ of tumor cells stained;,$++ 10-50 \%$; and +++ , more than $50 \%$ tumor cells stained positive. Both intensity and percentage of staining with 0 or + were considered as negative cases, while both intensity and percentage of staining with ++ and +++ were considered as positive cases.

\section{Cell lines and culture}

The human breast cancer cell lines MCF-7, MDA-MB231, T47D, SK-Br-3, ZR75-1, and MDA-MB-435 were obtained from ATCC (Manassas, VA) and grown in Dulbecco's modified Eagle's minimal essential medium (DMEM), supplemented with $10 \%$ fetal bovine serum, at $37^{\circ} \mathrm{C}$ in a humidified atmosphere of $95 \%$ air and $5 \% \mathrm{CO}_{2}$. Esophageal cancer TE-8, the stably RAR- $\beta_{2}-$ transfected TE-8S22, stably RRIG1-transfected TE-8RRIG1 cells, and prostate cancer PC3 cells were from our previous study [5]. These cell lines were grown in Dulbecco's modified Eagle's minimal essential medium (DMEM), supplemented with $10 \%$ fetal bovine serum, at $37^{\circ} \mathrm{C}$ in a humidified atmosphere of $95 \%$ air and $5 \%$ $\mathrm{CO}_{2}$. The growth medium for the stable gene transfected cells also contained $200 \mu \mathrm{g} / \mathrm{ml}$ G418 (Invitrogen, Carlsbad, CA).

\section{RRIG1 expression vector and transient gene transfection}

RRIG1 sense and antisense open-reading frames were inserted into pCDNA3.1 plasmid as reported in our previous studies $[5,6]$. The plasmids were used for transient transfection into MDA-MB-231, MDA-MB-435, and T47D with FuGENE 6 (Roche Diagnostics, Indianapolis, IN) or Lipofectamine ${ }^{\mathrm{TM}} 2000$ (Invitrogen). Thirty-six hours after the transfection, the cells were selected with $1200 \mu \mathrm{g} / \mathrm{mL}$ G418 (Invitrogen) for 5 days. The total cellular protein was extracted from the cells and subjected to Western blotting analysis. In duplicate experiments, RNA was extracted from the cells and subjected to semiquantitative reverse transcription-polymerase chain reaction (RT-PCR) analysis of gene expression as 
described previously [6,7]. After preliminary investigation to determine the optimal number of PCR cycles (between 25 and 34), we used 32 cycles of PCR to amplify RRIG1 mRNA. GAPDH was used as a loading control. The primers for RRIG1 mRNA were 5'CTCCCAGGGTGCCATATTT-3' and 5'-GTCATAGAGCACCCGAGCTT-3', which generated a 211-bp band. The primers for MMP-9 expression were 5'GCACGACGTCTTCCAGTACC-3' and 5'-GTTTGTAT CCGGCAAACTGG-3', which generated a 224-bp band. The primers for SH3GLB2 expression were 5'-GCAGACAGCACCAAGAACTG-3' and 5'-TTTTCAGCTT CTGCCACCTT-3', which generated a 233-bp band. GAPDH primers were 5'-CCCTTCATTGACCTCAACTACATGG-3' and 5'-CATGGTGGTGAAGACGCCAG3', which generated a 192-bp band.

\section{MTT assay}

The cells were grown and transiently transfected with RRIG1 sense or antisense cDNA and then grown in G418-containing medium for 1 or 5 days. For the methyl thiazolyl tetrazolium (MTT) assay, $20 \mu \mathrm{L}$ of MTT (5 mg/ml, Sigma, St. Louis, MO) was added to each well of the 96-well plates and incubated for an additional $4 \mathrm{~h}$. After the growth medium was removed, $100 \mu \mathrm{L}$ of dimethyl sulfoxide was added to the wells to dissolve the MTT crystal, and the optical densities were measured with an automated spectrophotometric plate reader at a single wavelength of $540 \mathrm{~nm}$. The percentage of cell growth was calculated using the formula: \% control $=$ ODt $/$ ODc $\times 100$, where ODt and ODc are the optical densities for transfected and vector control cells, respectively.

\section{Colony formation assay}

The ability of cells to form colonies in soft agarose is indicative of anchorage independence and is used as an in vitro criterion of transformation. We examined whether these cells can form colonies in soft agar after gene transfection [9]. Briefly, $2 \times 10^{3}$ gene-transfected cells were mixed in low-temperature-melting agarose $(0.35 \%)$ and then placed on top of solidified agarose in 60-mm-diameter dishes. After the top agarose with cells solidified in the cold room for $15 \mathrm{~min}$, the dishes were incubated in a humidified atmosphere of $95 \%$ air and $5 \% \mathrm{CO}_{2}$ at $37^{\circ} \mathrm{C}$ for 21 days. At the end of the experiments, the colonies were visualized by incubation with MTT at $37^{\circ} \mathrm{C}$ for $4 \mathrm{~h}$ and counted under an inverted microscope at $40 \times$ magnification.

\section{Migration and invasion assay}

Boyden chambers coated with and without Matrigel (obtained from BD Biosciences, Bedford, MA) were used for assaying tumor cell invasion and migration ability, respectively [5]. The transfected cells were first starved in medium without fetal calf serum (FCS) overnight, and the cells $\left(5 \times 10^{4}\right)$ were resuspended in the FCSfree medium and put into the top chambers in triplicate. The lower chamber was filled with $10 \%$ FCS as the chemoattractant and incubated for approximately $24 \mathrm{~h}$ for the migration assay and $48 \mathrm{~h}$ for the invasion assay. The surface was then wiped with a cotton swab to remove the cells on the upper surface. The cells that invaded the Matrigel and attached to the lower surface of the filter were fixed and stained with $1 \%$ crystal violet solution. The membranes with or without Matrigel were then gently removed from the chamber and mounted on glass slides. Six microscopic fields (at 100× magnification) per chamber were photographed. The cells in the photographs were then counted, and the data were summarized as means \pm standard deviation and presented as the percentage of control. Student $t$-test was used to determine statistical differences between the control and RRIG1 sense- or antisense-transfected breast cancer cells.

\section{Protein extraction and western blotting}

Total cellular protein was isolated from the gene-transfected breast cancer cells as described previously [5-7]. The protein concentration was then measured with a Bio-Rad Protein Assay Kit II (BioRad Laboratories, Hercules, CA) according to the manufacturer's protocol. Samples containing $50 \mu \mathrm{g}$ of protein from control or treated cells were separated by $10-14 \%$ polyacylamide sodium dodecyl sulfate-polyacrylamide gel electrophoresis gels and then transferred electrophoretically to a Hybond-C nitrocellulose membrane (GE-Healthcare, Arlington Heights, IL) at $500 \mathrm{~mA}$ for $2 \mathrm{~h}$ at $4{ }^{\circ} \mathrm{C}$. The membrane was subsequently stained with $0.5 \%$ Ponceau $\mathrm{S}$ (Sigma) containing $1 \%$ acetic acid to confirm that proteins were loaded equally and to verify transfer efficiency. The membranes were next incubated overnight in a blocking solution containing $5 \%$ bovine skim milk and $0.1 \%$ Tween 20 in PBS at $4^{\circ} \mathrm{C}$. The next day, the membranes were incubated with primary antibodies for $2 \mathrm{~h}$ at room temperature. The antibodies used were anti-p-Erk1/2, t-Erk1/2, p-Akt, t-Akt, p-Stat3, t-Stat3, p$\mathrm{Rb}$, and $\mathrm{Rb}$ (Cell Signaling Technology, Beverly, MA); anti-c-Jun and E2F-1 (Santa Cruz Biotechnology, Santa Cruz, CA); and anti- $\beta$-actin (Sigma). The membranes were washed in PBS and incubated for $1.5 \mathrm{~h}$ with horseanti-mouse or goat-anti-rabbit secondary antibody (GEHealthcare) diluted 1:5000. The membranes were then incubated with enhanced chemiluminescence solution (GE-Healthcare) for 1-2 min and exposed to X-ray film. The target band of proteins from western blots was quantified by using NIH ImageJ 1.34 s software $(\mathrm{NIH}$, Bethesda, MD) and normalized to $\beta$-actin. After that, 
the percentage of control was calculated using the formula: value of the RRIG1 sense or antisense transfected cells/value of the vector-only transfected cells.

\section{RhoA activation assay}

The RRIG1 sense and antisense cDNA-transfected breast cancer cells were detached with $0.05 \%$ trypsin, counted, reseeded, and cultured in new dishes in DMEM with $10 \%$ FCS for 16 hours and then in DMEM without FCS for an additional 12 hours. To activate RhoA, the cells were cultured in DMEM with 10\% FCS for $6 \mathrm{~h}$, and the total cellular protein was extracted in an ice-cold lysis buffer containing $20 \mathrm{mM}$ Tris- $\mathrm{HCl}(\mathrm{pH}$ 7.5), $10 \mathrm{mM} \mathrm{MgCl}, 150 \mathrm{mM} \mathrm{NaCl}, 1 \mathrm{mM} \mathrm{Na}{ }_{2}$ EDTA, 1 mM EGTA, 1\% Triton X-100, $2.5 \mathrm{mM}$ sodium pyrophosphate, $1 \mathrm{mM} \beta$-glycerophosphate, $1 \mathrm{mM} \mathrm{Na}_{3} \mathrm{VO}_{4}$, and $1 \mu \mathrm{g} / \mathrm{ml}$ leupeptin. Activated GTP-bound Rho protein in the cell lysates was pulled down using a recombinant GST-tagged Rhotekin Rho-binding domain (Millipore, Billerica, MA) and analyzed in western blots using antiRhoA antibody (Santa Cruz Biotechnology). Levels of the activated RhoA protein were normalized with the total cell lysates that were not subjected to the pulldown assay.

\section{Results}

Immunohistochemical analysis of RRIG1 expression in breast tissue specimens

In this study, we first verified the specificity of our rabbit polyclonal anti-RRIG1 antibody using three different experiments. Briefly, we first performed western blot with this antibody to detect expression of RRIG1 protein in vitro translated with an in vitro translation kit from Promega (Madison, WI). The data showed that this antibody specifically recognized RRIG1 protein (Additional Figure S1a). Meanwhile, in two different cancer cell lines we detected RRIG1 expression, which had been established by Northern blot in our previous study. We found that RRIG1-positive esophageal cancer cell line TE-8S22 expressed RRIG1 protein, whereas the PC3 cells did not show the target band, although the western blot showed a number of nonspecific bands (Additional Figure S1b), indicating that this antibody is not suitable for western blot assay.

To determine whether this antibody could be used for immunohistochemistry, we compared the data between immunohistochemistry and in situ hybridization for expression of RRIG1 mRNA and protein in the matched cells and tissues (Additional Figure S1cd). These data suggest that this antibody is suitable for immunohistochemistry. Next, we used immunohistochemical analysis to determine the RRIG1 expression in breast tissue specimens and found that RRIG1 was expressed in 14 of 15 normal mammary glands, 8 of 9 cases of atypical hyperplasia of the mammary gland, 6 of 10 ductal carcinoma in situ tissues, and 50 of 77 invasive breast cancer tissues $(P=0.023$ between normal and invasive cancer by Fisher exact test) (Figure 1).

Finally, we associated RRIG1 expression with the clinicopathologic features of the breast cancer patients and found a statistically significant reverse correlation between RRIG1 expression and lymph node metastasis in breast cancer (Table 1).

RRIG1 regulation of breast cancer cell growth, migration, and invasion

We then detected expression of RRIG1 mRNA in 6 different breast cancer cell lines using semiquantitative RTPCR and found that MDA-MB-435 and T47D expressed high levels of RRIG1 mRNA and that MCF-7 and SK-Br3 expressed low levels of RRIG1 mRNA. In contrast, MDA-MB-231 and ZR75-1 did not express RRIG1 mRNA (Figure 2A). Given these findings, we chose MDA-MB-231 and MDA-MB-435 for modulation of RRIG1 expression to assess the changed cell behaviors and gene expression. We transiently transfected RRIG1 sense and antisense cDNAs into the MDA-MB-231 and MDA-MB-435 cell lines, respectively. After gene transfection, RRIG1 expression was knocked down by RRIG1 antisense vector in MDA-MB-435 cells, while RRIG1 cDNA transfection restored RRIG1 expression in MDAMB-231 cells (Figure 2B). Next, we used the MTT assay to detect the changed cell viability by RRIG1 and found that restoration of RRIG1 expression reduced cell viability in MDA-MB-231 cells, but knockdown of RRIG1 expression induced cell viability in MDA-MB-435 cells after $5 \mathrm{~d}$ of cultures (Figure 2C). Furthermore, RRIG1 expression decreased the number of colonies in MDAMB-231 cells, and knockdown of RRIG1 expression in MDA-MB-435 cells increased the numbers of colonies in soft agar (Figure 2D). In addition, our data also showed that RRIG1 decreased migration and invasion capacity of MDA-MB-231 cells but increased the migratory and invasion capacity of MDA-MB-435 cells (Figure 3).

RRIG1 regulation of gene expression in breast cancer cells To determine the underlying molecular events of RRIG1 in the inhibition of breast cancer cell proliferation, migration and invasion, we performed semiquantitative RT-PCR and western blotting analysis to assess RRIG1 regulation of gene expression and the RhoA activation assay to detect the changed RhoA activity. We found that RRIG1 expression downregulated p-Erk1/2, p-AKT, total AKT, c-Jun, and MMP9 expression in MDA-MB-231 cells, whereas knockdown of RRIG1 expression upregulated p-AKT, total AKT, p-Stat3, p-RB, and E2F-1 expression in MDAMBN-435 cells (Figure 4). However, these data demonstrated that RRIG1 sense and antisense cDNAs regulated 

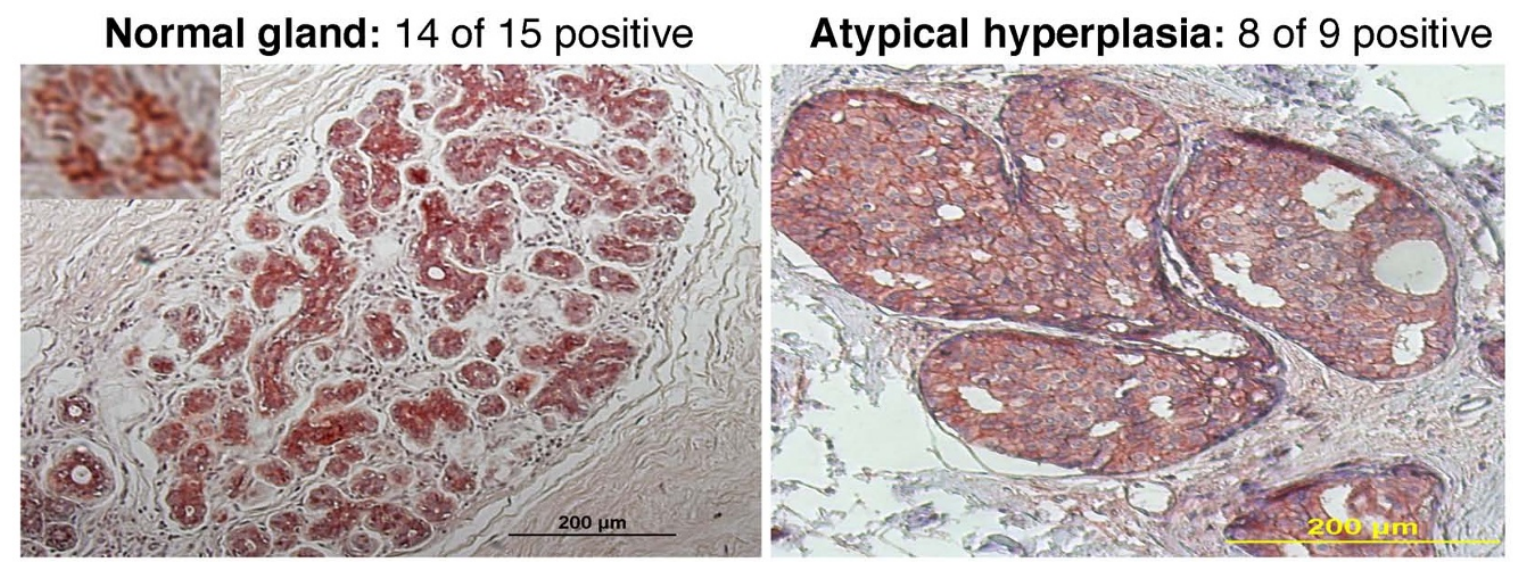

DCIS: 6 of 10 positive
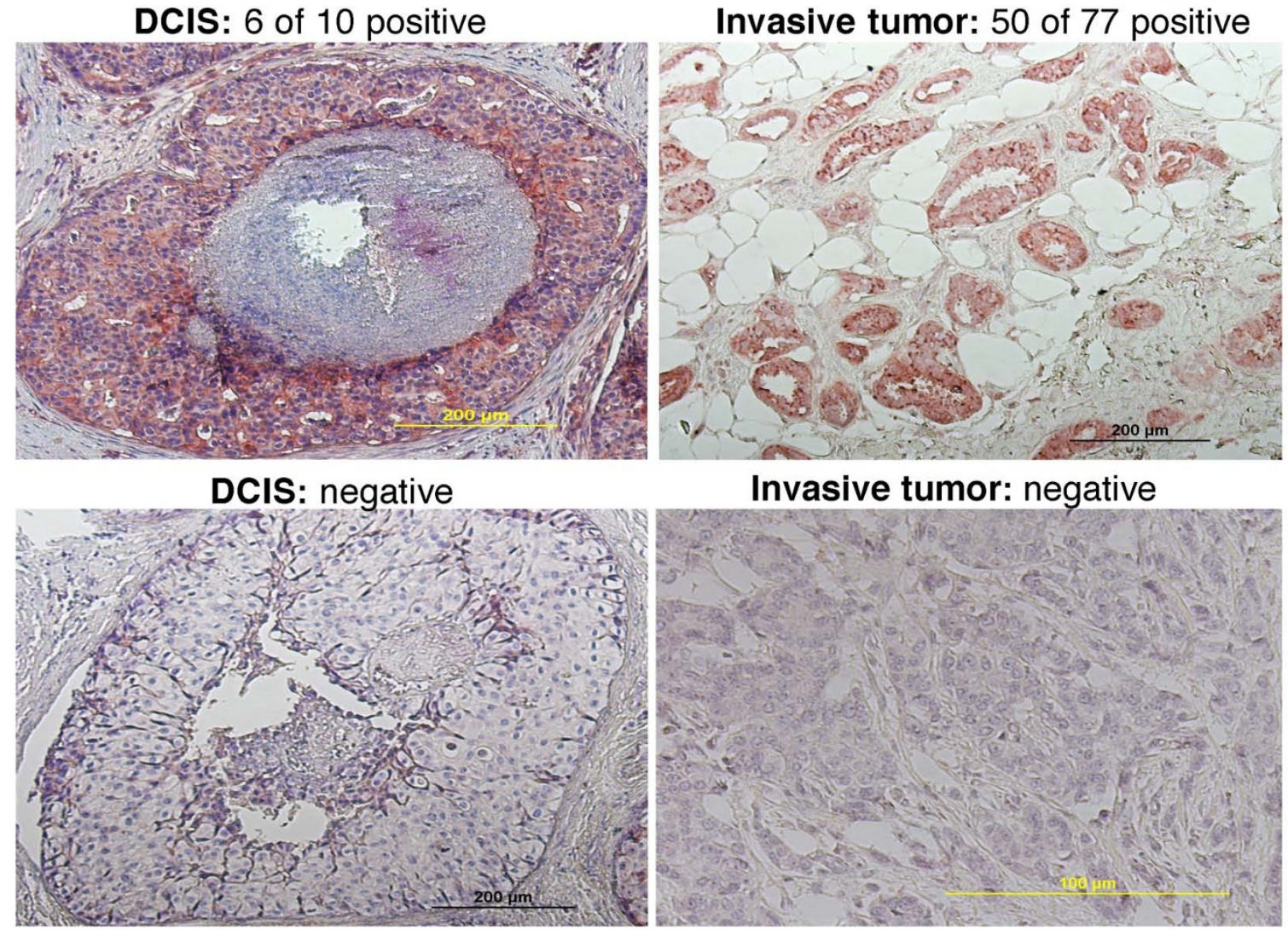

Invasive tumor: negative

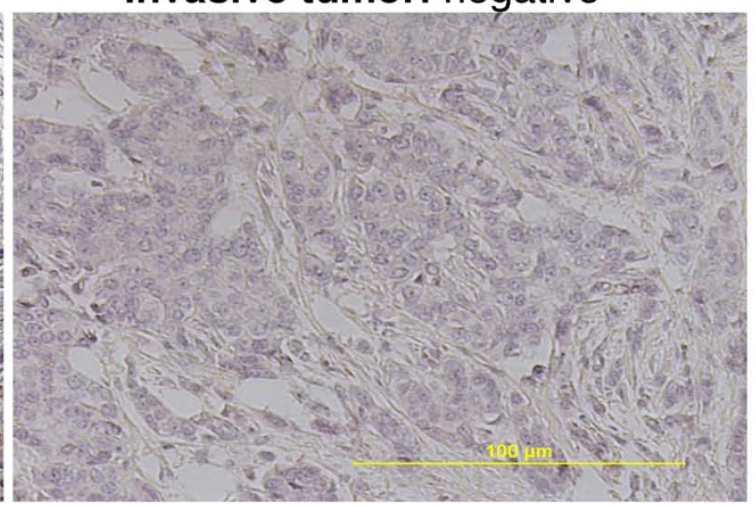

Figure 1 Immunohistochemical analysis of RRIG1 expression. Breast tissue sections were immunostained with the rabbit polyclonal antiRRIG1 antibody. DCIS, ductal carcinoma in situ.

expression of different genes, indicating that different breast cancer cell lines may have different gene alterations and that RRIG1 can regulate expression of some of them for control of breast cancer cell proliferation, migration, and invasion. Furthermore, we confirmed that RRIG1 was able to suppress RhoA activity, whereas antisense RRIG1 promoted RhoA activity in these breast cancer cells (Figure 4C). Because a recent study reported that the
MDA-MB-435 cell line may have originated from melanoma cells $[10,11]$, we added another RRIG1-positive cell line for antisense RRIG1 transfection. Antisense RRIG1 cDNA reduced RRIG1 mRNA levels but did not affect expression of SH3 domain GRB2-like endophilin B2 (SH3GLB2) (Figure 5). Knockdown of RRIG1 expression enhanced cell viability and invasion capacity of T47D cells (Figure 5). 
Table 1 Association of RRIG1 expression with clinicopathologic features of breast cancer patients

\begin{tabular}{|c|c|c|c|}
\hline \multirow[t]{2}{*}{ Variable } & \multicolumn{2}{|c|}{ RRIG1 expression } & \multirow[b]{2}{*}{ p Value } \\
\hline & Positive & Negative & \\
\hline Patient age (years) & & & 0.30 \\
\hline$\leq 35$ & 3 & 0 & \\
\hline $35-55$ & 34 & 17 & \\
\hline$>55$ & 13 & 10 & \\
\hline Tumor size (cm) & & & 0.66 \\
\hline$\leq 2$ & 8 & 3 & \\
\hline $2-5$ & 32 & 20 & \\
\hline$>5$ & 10 & 4 & \\
\hline Lymph node metastasis & & & 0.01 \\
\hline+ & 22 & 20 & \\
\hline- & 28 & 7 & \\
\hline Tumor differentiation & & & 0.18 \\
\hline 1 & 16 & 5 & \\
\hline$\|$ & 27 & 14 & \\
\hline III & 7 & 8 & \\
\hline Estrogen receptor & & & 0.64 \\
\hline+ & 25 & 15 & \\
\hline- & 25 & 12 & \\
\hline \multicolumn{4}{|l|}{ Progesterone receptor } \\
\hline+ & 27 & 15 & 0.89 \\
\hline- & 23 & 12 & \\
\hline c-erbB-2 & & & 0.62 \\
\hline+ & 14 & 9 & \\
\hline- & 36 & 18 & \\
\hline
\end{tabular}

\section{Discussion}

In the current study, we analyzed RRIG1 expression in breast tissue specimens and then determined the role of RRIG1 in breast cancer cells. We found that RRIG1 expression is reduced in breast cancer tissues, as reported by us previously [7]. RRIG1 expression was inversely correlated with lymph node metastasis of breast cancer. Furthermore, the restoration of RRIG1 expression in a breast cancer cell line resulted in tumorsuppressor activities, such as inhibition of tumor cell proliferation, colony formation, migration, and invasion. At molecular levels, RRIG1 suppressed expression of p-Erk1/2, p-Akt, total Akt, c-Jun, and MMP9 and RhoA activity. These genes are related to cell growth, adhesion, and mobility. However, knockdown of RRIG1 expression in another breast cancer cell line promoted tumor cell proliferation, colony formation, migration, and invasion, but these effects were associated with regulation of p-Stat3, p-Rb, and E2F-1.This study suggests that RRIG1 plays a role in suppressing breast cancer progression or even development of breast cancer.

Our previous studies [5-7] demonstrated that RRIG1 is a novel gene and may be a putative tumor suppressor. RRIG1 gene contains 6 exons, with exons 2 , 3, and 5 shared with exons 8, 9, and 10 of SH3GLB2 cDNA [7]. However, the open reading frames of these two genes are different, and therefore they are different genes. Moreover, our study also revealed that these two genes were differentially expressed in esophageal and prostate cancer cell lines $[5,8]$ and that benzo[a]pyrene diol epoxide (BPDE) reduced RRIG1 expression but did not alter SH3GLB2 mRNA levels in esophageal cancer cell lines (our unpublished data). These findings indicate that RRIG1 is a novel protein with no similarities to other proteins and that RRIG1 protein contains several putative functional motifs, such as a cadherin signaturelike motif, a glycoprotein GG/GX motif, and prolinerich regions that contain $\mathrm{SH} 3$ domain-binding motifs (PxxP). The latter motif was able to functionally mediate RRIG1 antitumor activity by suppressing tumor cell viability and cyclin D1 expression [7]. Nevertheless, it is unclear whether knockdown of RRIG1 expression using a vector carrying antisense open reading frame of RRIG1 can block expression of SH3GLB2 protein (Because no anti-SH3GLB2 antibody is available, we could not perform such an experiment). However, our current study showed that the vector carrying antisense open reading frame of RRIG1 did not affect expression of SH3GLB2 mRNA (Figure 5).

Our current and previous studies clearly demonstrated that reduced or lost expression of RRIG1 is an important event in the development or progression of human cancers, although the mechanism underlying the loss of expression of the RRIG1 gene remains unknown [5-8]. Moreover, our previous studies [12-14] reported that benzo $[a]$ pyrene diol epoxide (BPDE), a carcinogen present in tobacco smoke and environmental pollution, as well as bile acid, a tumor promoter in the gastrointestinal tract, inhibited RRIG1 expression, whereas retinoic acid induced RRIG1 expression, which was associated with downregulation of RAR- $\beta_{2}$ expression by these carcinogens. In addition, previous studies demonstrated a loss of RAR- $\beta_{2}$ expression in breast cancer tissues and cells [15-17]. Further study will be needed to determine the molecular mechanism that contributes to the loss of RRIG1 expression and, most importantly, to define RRIG1 as a tumor-suppressor gene in the inhibition of tumor cell growth, invasion, and gene expression.

Our current study demonstrated that the restoration ofRRIG1 expression inhibited breast cancer cell proliferation,colony formation, migration, and invasion and regulated geneexpression. Inhibition of phosphorylated Erk1/2 and Akt and c-Junexpression by RRIG1 may mediate RRIG1 suppression of cell growth, while reduced MMP9 expression by RRIG1 mediates the reduced tumor cell mobility and invasion capacity. Our previous study showed that MMP9 was upregulated in breast cancer [18]. Erk1/2 and Akt proteins play an 


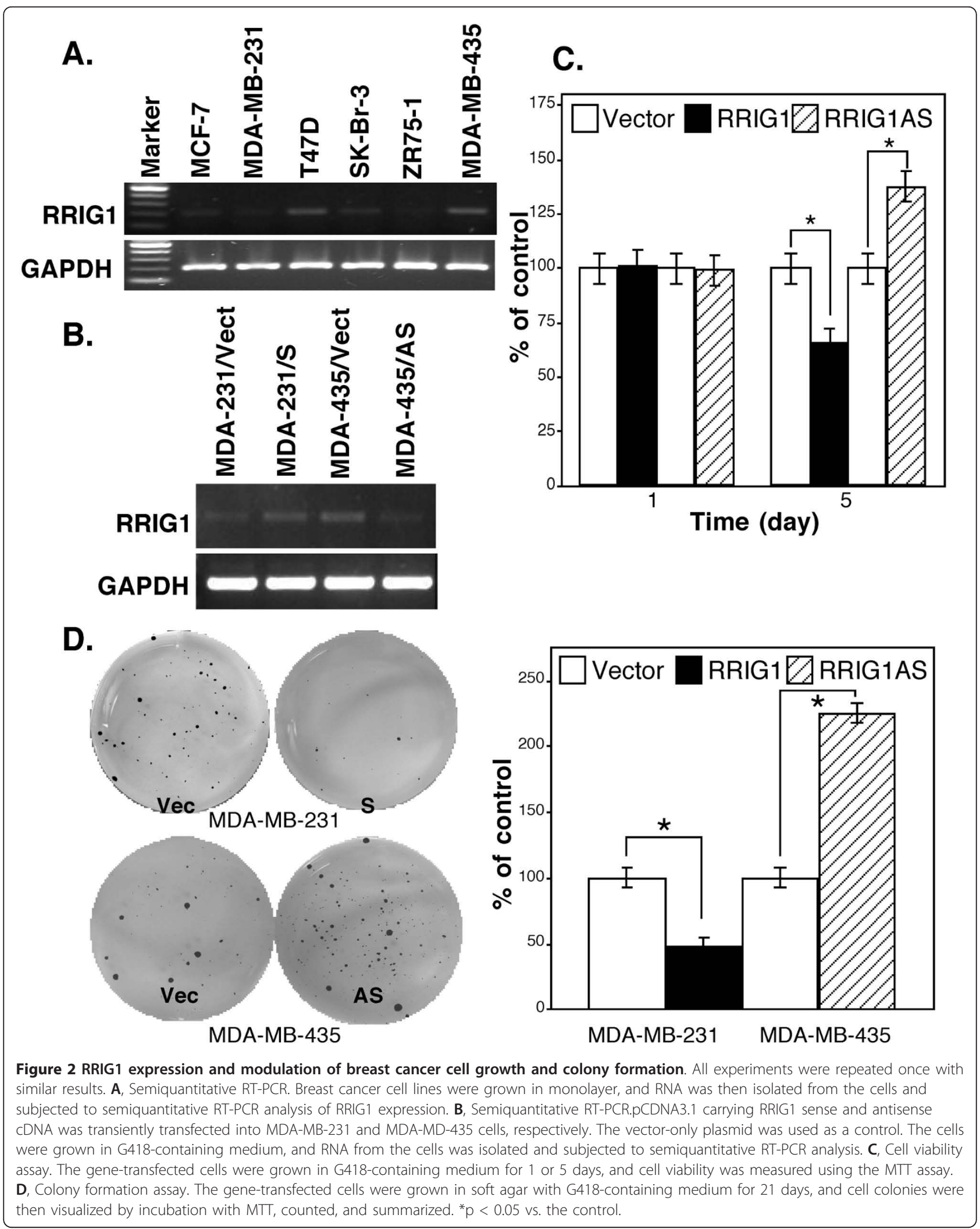




\section{A. Migration}
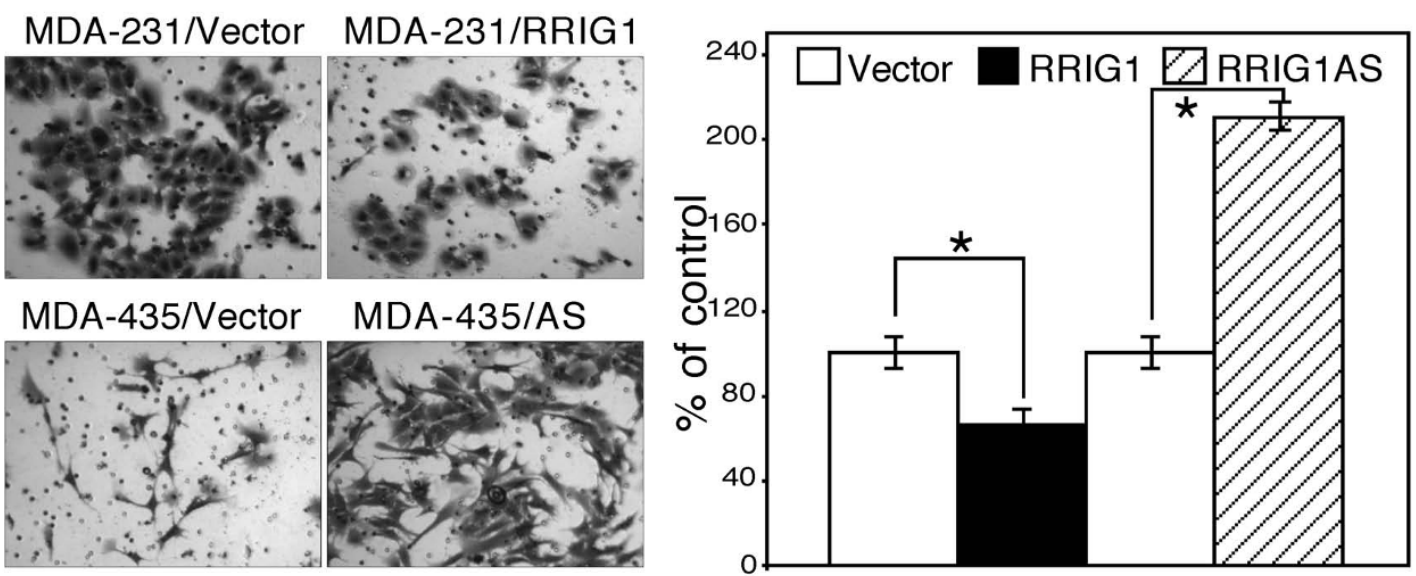

\section{B. Invasion}
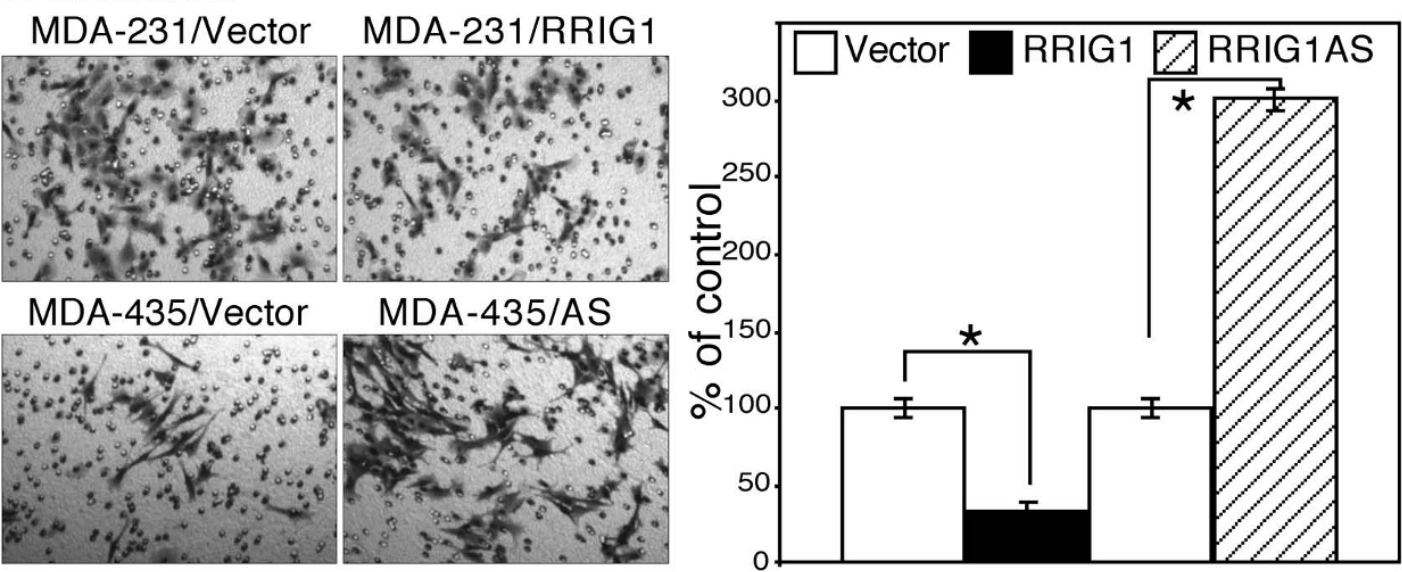

Figure 3 Tumor cell migration and invasion assays. All experiments were performed in triplicate and repeated twice. A, Migration assay. The RRIG1 sense and antisense-transfected MDA-MB-231 and MDA-MB-435 cells, respectively, were grown in G418-containing medium and then subjected to cell migration assay in Boyden chambers without Matrigel for $24 \mathrm{~h}$. The cells that migrated were stained with $1 \% \mathrm{crystal}$ violet solution and then counted and summarized. B, Invasion assay. These gene-transfected breast cancer cells were grown in G418-containing medium and then subjected to the cell invasion assay in Boyden chambers with Matrigel for $48 \mathrm{~h}$. The invaded cells were stained with $1 \%$ crystal violet solution and then counted and summarized. ${ }^{*} p<0.05$ vs. the control.

important role in cell survival, and activation of Akt has been shown to overcome cell cycle arrest in G1 and G2 phases of the cell cycle to enable cell proliferation [19-22]. Nevertheless, knockdown of RRIG1 expression has had opposite effects in the regulation of breast cancer cell growth, colony formation, migration, and invasion. Interestingly, these effects were through RRIG1 regulation of some other gene expressions, such as phosphorylated Stat3 and Rb. A previous study has shown the retinoic acid suppressed Stat3 phosphorylation in skin cancers [23]. Stat3 is a transcriptional factor and mediates expression of a variety of genes in response to cell stimuli and thus plays a key role in many cellular processes such as cell growth and apoptosis [24,25]. RRIG1 protein contains two putative Src homology 3 (SH3) domain-binding motifs that were characterized in a previous study [26], and our preliminary data showed that RRIG1 was able to bind to Src protein [8]. Previous studies [see reviews in ref. [25]] demonstrated that the role of STAT3 as a downstream signal transducer in Src family kinase-mediated tumorigenesis suggests that RRIG1 may suppress Stat3 activity through binding to Src protein. However, this needs further investigation.

Furthermore, our current study also confirmed our previous finding that RRIG1 inhibits RhoA activation to execute some of its biological functions [5]. RhoA is a small GTPase protein known to regulate the actin cytoskeleton in controlling cell mobility and growth and modulating gene expression [27-31]. The aberrant activation of RhoA proteins was found to cause cell growth, transformation, invasion, and metastasis in experimental models of carcinogenesis, and inhibition of RhoA 


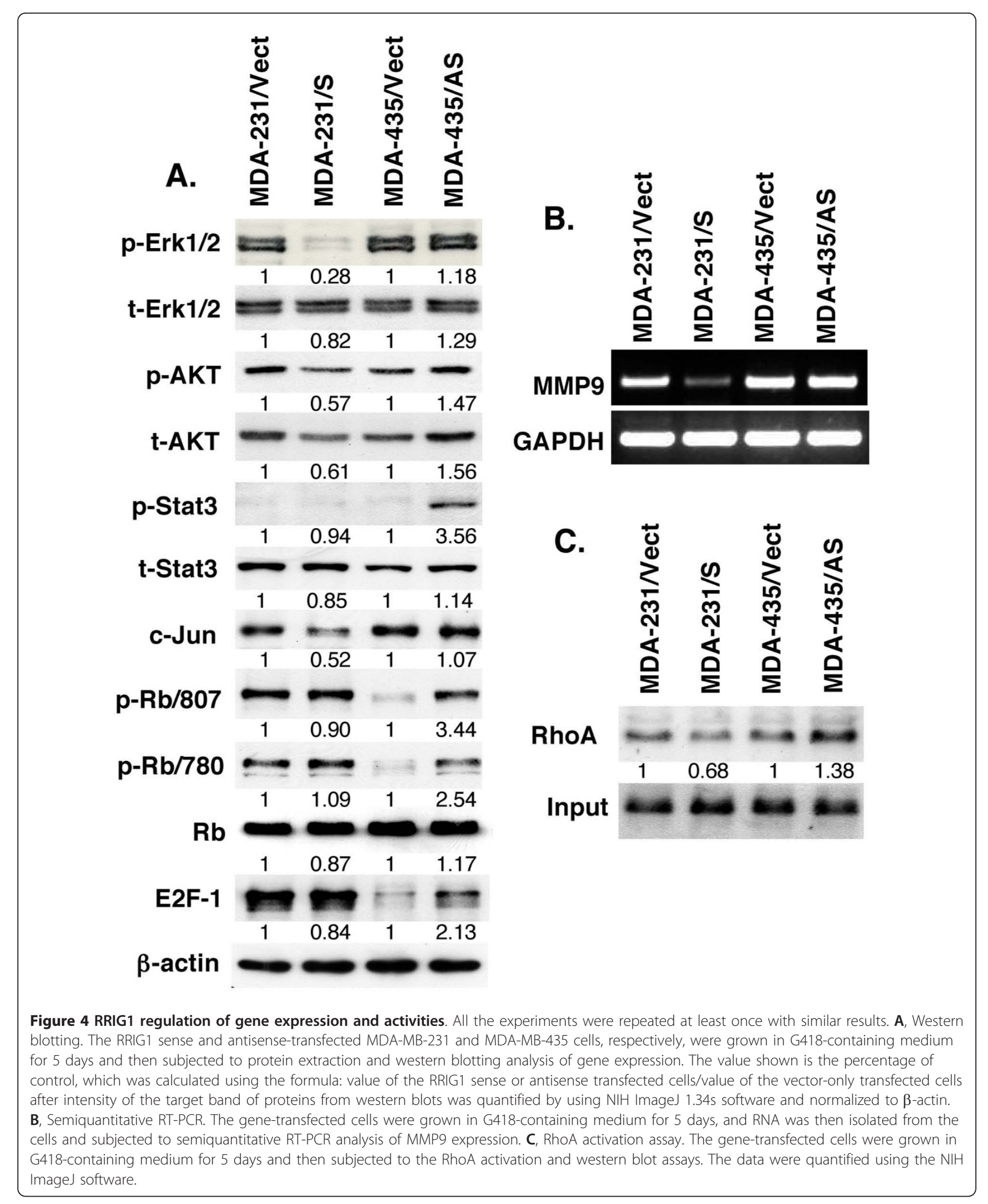




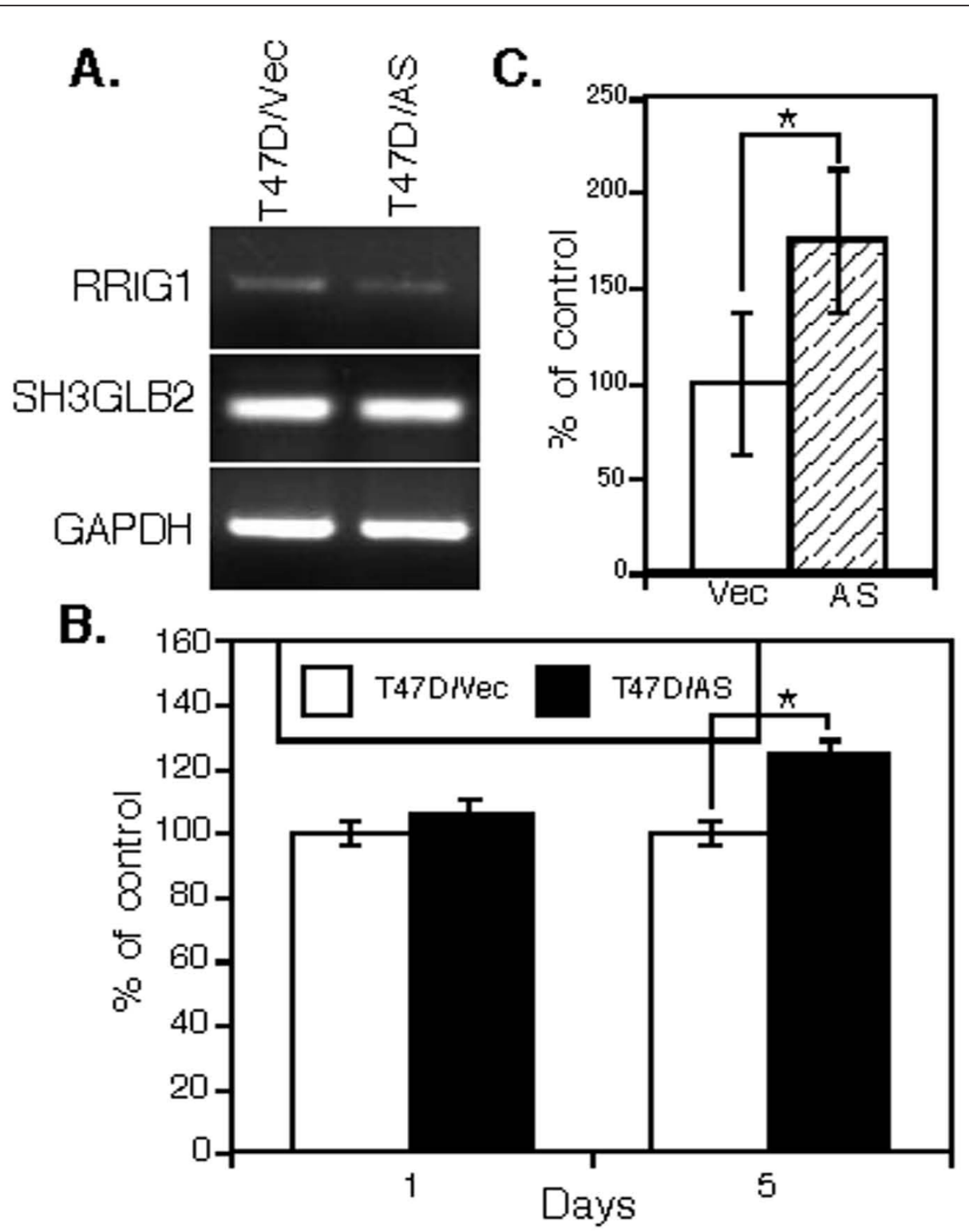

Figure 5 The effects of antisense RRIG1 in breast cancer T47D cells. All experiments were repeated at least once with similar results. A, Semiquantitative RT-PCR. The gene-transfected cells were grown in G418-containing medium for 5 days, and RNA was then isolated from the cells and subjected to semiquantitative RT-PCR analysis of RRIG1, SH3GLB2, and GAPDH expression. B, MTT assay. The gene-transfected cells were grown in G418-containing medium for 1 or 5 days, and cell viability was measured using the MTT assay. C, Invasion assay. These genetransfected breast cancer cells were grown in G418-containing medium and then subjected to the cell invasion assay in Boyden chambers with Matrigel for $48 \mathrm{~h}$. The invaded cells were stained with $1 \%$ crystal violet solution and then counted and summarized. Vec, vector-only; AS, antisense RRIG1 cDNA; * $p<0.05$ vs. the control.

suppressed cell proliferation, invasion, and angiogenesis in vitro and in vivo [27-31]. The RRIG1 protein can bind to the RhoA protein, although it is unknown whether this binding is direct or indirect [5]. We studied the RRIG1 and RhoA association because, in our unpublished findings of an in vitro pull-down assay, RRIG1 protein was able to pull down RhoA protein.

\section{Conclusion}

Our current study clearly showed the importance of lower RRIG1 expression in breast cancer cell growth, colony formation, invasion, and altered gene expression. Future study will attempt to determine the underlying molecular mechanisms and define RRIG1 as a tumorsuppressor gene in breast cancer. 


\section{Additional material}

Additional File 1: Figure S1: Specificity of rabbit polyclonal antiRRIG1 antibody for immunohistochemistry. A, Western blot. RRIG1 protein was first in vitro translated with pGEM and PCDNA3.1 vectorscarrying RRIG1 open-reading frame CDNA and an in vitro protein translation kit. The samples from the in vitro translation were subjected to western blot analysis of RRIG1 expression using our polyclonal antiRRIG1 antibody. NC, negative control. B, Western blot. Esophageal cancer TE-8S22 and prostate cancer PC3 cells were grown on monolayer for 3 days and total cellular protein was extracted and subjected to western blot with the polyclonal anti-RRIG1 antibody. The vector controlled in vitro translational sample was used for negative control. NC, negative control. NS, non-specific. C, Immunohistochemistry. RRIG-1-negative esophageal cancer cells TE-8 and RRIG-1 stably transfected TE-8-RRIG1 cells were grown in organotypic cultures for 14 days and the 3-D cell layers were subjected to tissue processing and immunohistochemistry with the polyclonal anti-RRIG1 antibody. D, Expression of RRIG1 mRNA and protein in breast tissues using in situ hybridization (ISH, see ref. 5 for the detailed methodology) and immunohistochemistry (IHC). Additional Method Organotypic culture. Esophageal cancer cell lines TE-8 and TE8-RRIG1 were seeded on to collagen gels prepared by using type I rattail collagen (Collaborative Biomedical Products, Bedford, MA), and incubated overnight until the cells will be confluent. The gels were then elevated to the air-liquid interface by placing them onto a surgical stainless steel mesh platform. DMEM was added until it reached the undersurface of the collagen gel but not cover the cell layer. The cultures were incubated at $37^{\circ} \mathrm{C}$ for 2 weeks after elevation to the airmedium interface. In the end of the experiments, the cell discs were fixed in $4 \%$ paraformaldehyde and embedded in paraffin following by sectioning and staining.

\section{Acknowledgements}

We thank the Department of Scientific Publication at MD Anderson Cancer Center for editing the manuscript. This study was supported in part by National Cancer Institute grants R01 CA 117895 and R01 CA078480 and an Institutional Research Grant from MD Anderson Cancer Center as well as grants from The Science Foundations of Education Department of Anhui Province, China (KJ2007B195) and The Youth Foundations of Education Department of Anhui Province, China (2007jq1075)

\section{Author details}

'Department of Clinical Cancer Prevention, The University of Texas M. D. Anderson Cancer Center, Houston, Texas 77030, USA. ${ }^{2}$ Department of Experimental Therapeutics, The University of Texas M. D. Anderson Cancer Center, Houston, Texas 77030, USA. ${ }^{3}$ Department of Pathology, Anhui Medical University, Hefei, Anhui, China.

\section{Authors' contributions}

$G Z$ performed most of the experiments. $A B$ helped in research design and immunohistochemistry. BG performed experiments for T47D cells. ZF provided breast cancer cell lines and contributed to the research design, critical comments, and writing. PHB provided financial support and contributed to the research design. XCX participated in the overall research design, data collection, and writing. All authors read and approved the final Manuscript.

\section{Competing interests}

The authors declare that they have no competing interests.

Received: 18 October 2010 Accepted: 25 January 2011

Published: 25 January 2011

\section{References}

1. Wu K, Zhang Y, Xu XC, Hill J, Celestino J, Kim HT, Mohsin SK, Hilsenbeck SG, Lamph WW, Bissonette R, Brown PH: The retinoid X receptor-selective retinoid, LGD1069, prevents the development of estrogen receptor-negative mammary tumors in transgenic mice. Cancer Res 2002, 62:6376-6380.
2. DeVita VT Jr, Hellman S, Rosenberg SA, (eds): Cancer: Principles and Practice of Oncology. Philadelphia and New York: Lippincott-Raven Publishers; 1997, 1541-1616.

3. Fidler I, Nicholson G: Concepts and mechanisms of breast cancer metastasis. In The Breast. Edited by: Bland K, Copeland E. Philadelphia: WB Saunders; 1991:395-408.

4. Fidler IJ: Molecular biology of cancer: invasion and metastasis. In Cancer: Principles and Practice of Oncology. Edited by: DeVita VT Jr, Hellman S, Rosenberg SA. Philadelphia and New York: Lippincott-Raven Publishers; 1997:135-152.

5. Liang ZD, Lippman $S M, W u T$, Lotan R, Xu XC: RRIG1 mediates effects of retinoic acid receptor- $\beta_{2}$ on tumor cell growth and gene expression through binding to and inhibiting RhoA. Cancer Res 2006, 66:7111-7118.

6. Huang J, Liang ZD, Wu TT, Hoque A, Chen H, Jiang Y, Zhang H, Xu XC: Tumor-suppressive effect of retinoid receptor-induced gene-1 (RRIG1) in esophageal cancer. Cancer Res 2007, 67:1589-1593.

7. Guan B, Li H, Chen Y, Hoque A, Xu X-C: Characterization of retinoid receptor-induced gene-1 gene and its relationship to SH3 domain GRB2like endophilin B2 gene. J Cancer Mol 2009, 5:15-19.

8. Hoque A, Chen H, Guan B, Xu X-C: Tumor-suppressive effect of RRIG1 in prostate cancer. Proc Amer Assoc Cancer Res 2009, 50:5334

9. Xu XC, Liu X, Tahara E, Lippman SM, Lotan R: Expression and upregulation of retinoic acid receptor- $\beta$ is associated with retinoid sensitivity and colony formation in esophageal cancer cell lines. Cancer Res 1999, 59:2477-2483.

10. Ross DT, Scherf U, Eisen MB, Perou CM, Rees C, Spellman P, lyer $V$, Jeffrey SS, Van de Rijn M, Waltham M, Pergamenschikov A, Lee JC, Lashkari D, Shalon D, Myers TG, Weinstein JN, Botstein D, Brown PO: Systematic variation in gene expression patterns in human cancer cell lines. Nat Genet 2000, 24:227-235.

11. Ellison G, Klinowska T, Westwood RF, Docter E, French T, Fox JC: Further evidence to support the melanocytic origin of MDA-MB-435. Mol Pathol 2002, 55:294-299.

12. Song S, Xu X-C: Effect of benzo[a]pyrene diol epoxide on expression of retinoic acid receptor-beta in immortalized esophageal epithelial cells and esophageal cancer cells. Biochem Biophys Res Commun 2001, 281:872-877.

13. Song S, Lippman SM, Zou Y, Ye X, Ajani JA, Xu X-C: Induction of cyclooxygenase-2 by benzo[a]pyrene diol epoxide through inhibition of retinoic acid receptor-b2 expression. Oncogene 2005, 24:8268-8276.

14. Xu X-C: Risk factors and gene expression in esophageal cancer. In Cancer Epidemiology, Vol. I, Host Susceptibility Factors. Edited by: Verma M. Humana Press; 2009:335-360.

15. Xu XC, Sneige N, Liu X, Nandagiri R, Lee JJ, Lukmanji F, Hortobagyi G, Lippman SM, Dhingra K, Lotan R: Progressive decrease in nuclear retinoic acid receptor beta messenger RNA level during breast carcinogenesis. Cancer Res 1997, 57:4992-4996.

16. Widschwendter MJ, Berger G, Daxenbichler E, Muller-Holzner A, Widschwendter A, Mayr A, Marth C, Zeimet AG: Loss of retinoic acid receptor beta expression in breast cancer and morphologically normal adjacent tissue but not in the normal breast tissue distant from the cancer. Cancer Res 1997, 57:4158-4161.

17. Seewaldt VL, Johnson BS, Parker MB, Collins SJ, Swisshelm K: Expression of retinoic acid receptor beta mediates retinoic acid-induced growth arrest and apoptosis in breast cancer cells. Cell Growth Differ 1995, 6:1077-1088.

18. Wu ZS, Wu Q, Yang JH, Wang HQ, Ding XD, Yang F, Xu X-C: Prognostic significance of MMP-9 and TIMP-1 expression in breast cancer. Int $J$ Cancer 2008, 122:2050-2056.

19. Ramaswamy S, Nakamura N, Vazquez F, Batt DB, Perera S, Roberts TM, Sellers WR: Regulation of G1 progression by the PTEN tumor suppressor protein is linked to inhibition of the phosphatidylinositol 3-kinase/Akt pathway. Proc Natl Acad Sci USA 1999, 96:2110-2115.

20. Song $G$, Ouyang $G$, Bao $S$ : The activation of Akt/PKB signaling pathway and cell survival. J Cell Mol Med 2005, 9:59-71.

21. Hoshino R, Chatani Y, Yamori T, Tsuruo T, Oka H, Yoshida O, Shimada Y, Arii S, Wada H, Fujimoto J, Kohno M: Constitutive activation of the 41-/43$\mathrm{kDa}$ mitogen-activated protein kinase signaling pathway in human tumors. Oncogene 1999, 18:813-822.

22. McCubrey JA, Steelman LS, Chappell WH, Abrams SL, Wong EW, Chang F, Lehmann B, Terrian DM, Milella M, Tafuri A, Stivala F, Libra M, Basecke J, Evangelisti C, Martelli AM, Franklin RA: Roles of the Raf/MEK/ERK pathway 
in cell growth, malignant transformation and drug resistance. BBA-MO/ Cancer Res 2007, 1773:1263-1284.

23. Syed Z, Cheepala SB, Gill JN, Stein J, Nathan CA, Digiovanni J, Batra V, Adegboyega P, Kleiner HE, Clifford JL: All-trans retinoic acid suppresses Stat3 signaling during skin carcinogenesis. Cancer Prev Res 2009, 2:903-911.

24. Yuan ZL, Guan YJ, Wang L, Wei W, Kane AB, Chin YE: Central role of the threonine residue within the $p+1$ loop of receptor tyrosine kinase in STAT3 constitutive phosphorylation in metastatic cancer cells. Mol Cell Biol 2004, 24:9390-9400.

25. Silva CM: Role of STATs as downstream signal transducers in Src family kinase-mediated tumorigenesis. Oncogene 2004, 23:8017-8023.

26. Alexandropoulos K, Cheng G, Baltimore D: Proline-rich sequences that bind to Src homology 3 domains with individual specificities. Proc Natl Acad Sci USA 1995, 92:3110-3114.

27. Sahai E, Marshall CJ: RHO-GTPases and cancer. Nat Rev Cancer 2002, 2:133-142.

28. Vial E, Sahai E, Marshall CJ: ERK-MAPK signaling coordinately regulates activity of Rac1 and RhoA for tumor cell motility. Cancer Cell 2003, 4:67-79.

29. Gomez del Pulgar T, Benitah SA, Valeron PF, Espina C, Lacal JC: Rho GTPase expression in tumorigenesis: evidence for a significant link. Bioessays 2005, 27:602-613.

30. Sahai E, Marshall CJ: Differing modes of tumor cell invasion have distinct requirements for Rho/ROCK signaling and extracellular proteolysis. Nat Cell Biol 2003, 5:711-719.

31. Yoshioka K, Nakamori S, Itoh K: Overexpression of small GTP-binding protein RhoA promotes invasion of tumor cells. Cancer Res 1999, 59:2004-2010.

\section{Pre-publication history}

The pre-publication history for this paper can be accessed here: http://www.biomedcentral.com/1471-2407/11/32/prepub

doi:10.1186/1471-2407-11-32

Cite this article as: Zhang et al: Tumor-suppressor activity of RRIG1 in breast cancer. BMC Cancer 2011 11:32.

\section{Submit your next manuscript to BioMed Central} and take full advantage of:

- Convenient online submission

- Thorough peer review

- No space constraints or color figure charges

- Immediate publication on acceptance

- Inclusion in PubMed, CAS, Scopus and Google Scholar

- Research which is freely available for redistribution

Submit your manuscript at www.biomedcentral.com/submit
C Biomed Central 